\title{
Hidden antiunitary symmetry behind "accidental" degeneracy and its protection of degeneracy
}

\author{
Jing-Min $\mathrm{Hou}^{1, *}$ and Wei Chen ${ }^{2}$ \\ ${ }^{1}$ School of Physics, Southeast University, Nanjing 211189, China \\ 2 College of Science, Nanjing University of Aeronautics and Astronautics, \\ Nanjing 210016, China \\ Corresponding author. E-mail:* jmhou@seu.edu.cn
}

\begin{abstract}
In quantum mechanics, accidental degeneracy refers to energy degeneracy that occurs coincidentally, without any protection by symmetry. Here, we prove a theorem stating that any two-fold degeneracy (accidental or not) in a quantum system is protected by a novel hidden symmetry, which can be expressed by an antiunitary operator with its square being -1 . In this sense, the so-called accidental degeneracy is not really accidental, and this actually implies a hidden antiunitary symmetry.
\end{abstract}

Keywords: accidental degeneracy, hidden symmetry, antiunitary symmetry PACS numbers: 03.65.Aa, 02.20.-a, 03.65.Fd

\section{Introduction}

Energy degeneracy in a quantum system is generally protected by a symmetry, such as point group or time-reversal symmetry. Nevertheless, it is believed that there exists a type of degeneracy called accidental degeneracy that does not need the protection of symmetry. Accidental degeneracy was discussed in the early days of quantum theory by von Neumann and Wigner[1. To ensure that two eigenenergies of a $2 \times 2$ Hamiltonian matrix are identical, three independent equations should be satisfied. Thus, three real tunable parameters can guarantee the existence of a two-fold degeneracy. This type of degeneracy is usually considered to be accidental. Later, Herring applied this theory to the energy bands of crystals and developed the theory of accidental band degeneracy[2].

Some degeneracies in quantum systems that were previously considered to be accidental were later found to be protected by a higher hidden symmetry 3 . For example, the accidental degeneracy in the Kepler problem[4], an isotropic harmonic oscillator [5], a free particle enclosed by an impenetrable cubic box [6, 7], and a quantum mechanical rigid rotor [8] can be explained by expanding the symmetry group. However, not all 
the accidental degeneracies in quantum systems can be explained by a larger symmetry group. Whether a hidden symmetry exists for any accidental degeneracy is still an open question. Thus, a general explanation of all the accidental degeneracies is desirable.

In this paper, we provide insight into the interpretation of accidental degeneracy in quantum systems. First, we prove a theorem stating that any two-fold degeneracy must be protected by a hidden antiunitary symmetry (HAS) having an operator with its square being -1 . We emphasize that this HAS having an operator with its square being -1 is necessary and sufficient for the protection of any two-fold degeneracy. From the theorem, we can infer that the so-called "accidental" degeneracy is not really accidental. In our previous works, we found some examples in two-dimensional lattices, where the HAS is responsible for the degeneracy at the Dirac points [9, 10].

Because our theory of HAS protection of energy degeneracy is applicable to any type of two-fold degeneracy, it is important to clarify its relationship and compatibility with other theories. Most non-accidental two-fold degeneracies are believed to be protected by the conventional symmetry group. For these degeneracies, we show that the HAS protection is the minimum constraint for the existence of a two-fold degeneracy and that the constraints of the conventional symmetry group may be redundant.

\section{Theorem}

First, we prove the following theorem: In a quantum system, any two-fold degeneracy must be protected by a symmetry that has an antiunitary operator with its square being -1 .

Proof-We first suppose that $H$ is the Hamiltonian of a system and has two distinct eigenstates $\left|\psi_{1}\right\rangle$ and $\left|\psi_{2}\right\rangle$ with the same eigenenergy $E$, i.e.,

$$
H\left|\psi_{i}\right\rangle=E\left|\psi_{i}\right\rangle
$$

where $i=1,2$. That is, the system has a two-fold degeneracy. We can always construct an operator as

$$
\Upsilon=\left(\left|\psi_{2}\right\rangle\left\langle\psi_{1}^{*}|-| \psi_{1}\right\rangle\left\langle\psi_{2}^{*}\right|\right) K
$$

where the star represents complex conjugation, and $K$ is the complex conjugation operator. Obviously, $\Upsilon$ is an antiunitary operator, and it is easy to verify that the square of the operator $\Upsilon$ is $\Upsilon^{2}=-1$. We can verify the following equations:

$$
\begin{aligned}
& \Upsilon\left|\psi_{1}\right\rangle=\left|\psi_{2}\right\rangle, \\
& \Upsilon\left|\psi_{2}\right\rangle=-\left|\psi_{1}\right\rangle .
\end{aligned}
$$

From Eqs. (11), (3), and (4), we have

$$
(\Upsilon H-H \Upsilon)\left|\psi_{i}\right\rangle=0
$$

Then, we obtain $[\Upsilon, H]=0$; namely, the system is invariant under the action of the operator $\Upsilon$. 
On the other hand, we show that if $H$ is invariant under the the action of the antiunitary operator $\Upsilon$ with $\Upsilon^{2}=-1$, the system must have a two-fold degeneracy. We assume that $\left|\psi_{1}\right\rangle$ is the eigenstate of the Hamiltonian $H$ and define

$$
\left|\psi_{2}\right\rangle=\Upsilon\left|\psi_{1}\right\rangle
$$

then $\left|\psi_{1}\right\rangle$ and $\left|\psi_{2}\right\rangle$ have the same eigenenergy and may be the same eigenstate up to a factor or orthogonal to each other. Because $\Upsilon^{2}=-1$ and Eq. (6) are satisfied, we have

$$
\Upsilon\left|\psi_{2}\right\rangle=-\left|\psi_{1}\right\rangle
$$

Suppose $\left|\tilde{\psi}_{1}\right\rangle=\Upsilon\left|\psi_{1}\right\rangle$ and $\left|\tilde{\psi}_{2}\right\rangle=\Upsilon\left|\psi_{2}\right\rangle$; then, for the antiunitary operator, we have

$$
\left\langle\tilde{\psi}_{1} \mid \tilde{\psi}_{2}\right\rangle=\left\langle\psi_{1} \mid \psi_{2}\right\rangle^{*}=\left\langle\psi_{2} \mid \psi_{1}\right\rangle
$$

Furthermore, from Eqs. (6]) and (7), we obtain

$$
\left\langle\tilde{\psi}_{1} \mid \tilde{\psi}_{2}\right\rangle=-\left\langle\psi_{2} \mid \psi_{1}\right\rangle
$$

Comparing Eq. (9) with Eq. (8), we arrive at $\left\langle\psi_{2} \mid \psi_{1}\right\rangle=0$; i.e., the two eigenstates are orthogonal to each other. Considering that they have the same eigenenergy, we conclude that there must exist a two-fold degeneracy.

On the basis of the above theorem, we infer that real accidental degeneracy does not exist in any system. The degeneracy is considered to be accidental only because the symmetry behind it, which indeed exists, has not been found. The so-called "accidental" degeneracy is generally obtained by fine-tuning the parameters to satisfy some conditions, which means that the parameters obey some constraints. Actually, these constraints are equivalent to a HAS invariance of the Hamiltonian, which will be discussed in detail in the next section.

\section{Example: a two-level system}

Now, we turn to a two-level quantum system with the Hamiltonian $H(\alpha)$, where $\alpha$ represents all of the tunable parameters. Without loss of generality, the Hamiltonian of the quantum system can always be written in the form

$$
\begin{aligned}
H(\alpha) & =h_{0}(\alpha) I_{2}+h_{x}(\alpha) \sigma_{x}+h_{y}(\alpha) \sigma_{y}+h_{z}(\alpha) \sigma_{z} \\
& =\left(\begin{array}{cc}
h_{0}(\alpha)+h_{z}(\alpha) & h_{x}(\alpha)-i h_{y}(\alpha) \\
h_{x}(\alpha)+i h_{y}(\alpha) & h_{0}(\alpha)-h_{z}(\alpha)
\end{array}\right)
\end{aligned}
$$

where $\sigma_{x, y, z}$ are the Pauli matrices, and $I_{2}$ is the $2 \times 2$ unit matrix; $h_{i}(\alpha),(i=0, x, y, z)$ are real functions of $\alpha$. The eigenenergies are $E_{ \pm}(\alpha)=h_{0}(\alpha) \pm \sqrt{h_{x}(\alpha)^{2}+h_{y}(\alpha)^{2}+h_{z}(\alpha)^{2}}$. By fine-tuning the parameters to intersect the energy levels, one may obtain an energy degeneracy for the parameter $\alpha=\alpha_{0}$, which is generally considered to be accidental. 
From the expression of the eigenenergies, we can conclude that if energy degeneracy occurs for $\alpha=\alpha_{0}, h_{x}, h_{y}$ and $h_{z}$ must be constrained as follows:

$$
\begin{aligned}
& h_{x}\left(\alpha_{0}\right)=0, \\
& h_{y}\left(\alpha_{0}\right)=0, \\
& h_{z}\left(\alpha_{0}\right)=0 .
\end{aligned}
$$

In the following, we will show that these constraints on the Hamiltonian are equivalent to a HAS having an operator with its square being -1 .

We assume that $\Upsilon$ is an antiunitary operator and $\Upsilon^{2}=-1$. We suppose that when the parameter $\alpha=\alpha_{0}$, the Hamiltonian $H\left(\alpha_{0}\right)$ is invariant under the action of $\Upsilon$; i.e., $\Upsilon H\left(\alpha_{0}\right) \Upsilon^{-1}=H\left(\alpha_{0}\right)$. The representation of $\Upsilon$ can be written as

$$
\Upsilon=\left(\begin{array}{cc}
0 & -1 \\
1 & 0
\end{array}\right) K
$$

Then, under the action of the operator $\Upsilon$, we have

$$
\Upsilon H\left(\alpha_{0}\right) \Upsilon^{-1}=\left(\begin{array}{cc}
h_{0}\left(\alpha_{0}\right)-h_{z}\left(\alpha_{0}\right) & -h_{x}\left(\alpha_{0}\right)+i h_{y}\left(\alpha_{0}\right) \\
-h_{x}\left(\alpha_{0}\right)-i h_{y}\left(\alpha_{0}\right) & h_{0}\left(\alpha_{0}\right)+h_{z}\left(\alpha_{0}\right)
\end{array}\right)
$$

Comparing Eqs. (10) and (15) in terms of the identity $\Upsilon H\left(\alpha_{0}\right) \Upsilon^{-1}=H\left(\alpha_{0}\right)$, we obtain $h_{x}\left(\alpha_{0}\right)=h_{y}\left(\alpha_{0}\right)=h_{z}\left(\alpha_{0}\right)=0$, which is just Eqs. (11), (12), and (13).

From the above analysis, we find that the constraints on the energy functionals for degeneracy imply a HAS having an operator with its square being -1 , which can protect the energy degeneracy.

\section{Relationship between the HAS protection and a group symmetry protection}

In the above theorem, we showed that any two-fold degeneracy must be protected by a HAS. If there is a two-fold degeneracy protected by another symmetry, for example, a point group, one may then ask what the relationship is between the point group and the HAS. In the following, we will answer this question in detail.

If a two-fold degeneracy is protected by a group, then this group must have at least a two-dimensional irreducible representation, which requires a non-abelian group. We suppose that $A$ and $B$ are representation matrices of a two-dimensional irreducible unitary representation of the non-abelian group and do not commute with each other; i.e., $[A, B] \neq 0$. The unitary representation matrices can always be written as

$$
\begin{aligned}
A & =\left(\begin{array}{cc}
a_{1}+i a_{2} & a_{3}+i a_{4} \\
-a_{3}+i a_{4} & a_{1}-i a_{2}
\end{array}\right) \\
B & =\left(\begin{array}{cc}
b_{1}+i b_{2} & b_{3}+i b_{4} \\
-b_{3}+i b_{4} & b_{1}-i b_{2}
\end{array}\right)
\end{aligned}
$$


where $a_{i}$ and $b_{i}(i=1,2,3,4)$ are real numbers and satisfy $\sum_{i=1}^{4}\left|a_{i}\right|^{2}=1$ and $\sum_{i=1}^{4}\left|b_{i}\right|^{2}=$ 1 , respectively. The commutation of the matrices $A$ and $B$ reads

$$
[A, B]=2\left(\begin{array}{cc}
i \lambda_{1} & -\lambda_{2}+i \lambda_{3} \\
\lambda_{2}+i \lambda_{3} & -i \lambda_{1}
\end{array}\right)
$$

where $\lambda_{1}=a_{3} b_{4}-a_{4} b_{3}, \lambda_{2}=a_{2} b_{4}-a_{4} b_{2}$, and $\lambda_{3}=a_{2} b_{3}-a_{3} b_{2}$. Because the matrices $A$ and $B$ do not commute with each other, at least one of $\lambda_{i}(i=1,2,3)$ is nonvanishing. Because the system is invariant under transformation of the non-abelian group, we have

$$
\begin{aligned}
& {\left[A, H\left(\alpha_{0}\right)\right]=0,} \\
& {\left[B, H\left(\alpha_{0}\right)\right]=0 .}
\end{aligned}
$$

On the other hand, from Eqs. (10) and (16), we have

$$
\left[A, H\left(\alpha_{0}\right)\right]=2\left(\begin{array}{cc}
W_{1}\left(\alpha_{0}\right) & W_{2}\left(\alpha_{0}\right)+i W_{3}\left(\alpha_{0}\right) \\
W_{2}\left(\alpha_{0}\right)-i W_{3}\left(\alpha_{0}\right) & -W_{1}\left(\alpha_{0}\right)
\end{array}\right)
$$

where $W_{1}\left(\alpha_{0}\right)=a_{3} h_{x}\left(\alpha_{0}\right)-a_{4} h_{y}\left(\alpha_{0}\right), W_{2}\left(\alpha_{0}\right)=a_{2} h_{y}\left(\alpha_{0}\right)-a_{3} h_{z}\left(\alpha_{0}\right)$, and $W_{3}\left(\alpha_{0}\right)=$ $2 a_{2} h_{x}\left(\alpha_{0}\right)-2 a_{4} h_{z}\left(\alpha_{0}\right)$. Comparing Eq. (21) with Eq. (19), we obtain the following three equations:

$$
\begin{aligned}
& a_{3} h_{x}\left(\alpha_{0}\right)-a_{4} h_{y}\left(\alpha_{0}\right)=0 \\
& a_{2} h_{y}\left(\alpha_{0}\right)-a_{3} h_{z}\left(\alpha_{0}\right)=0 \\
& a_{2} h_{x}\left(\alpha_{0}\right)-a_{4} h_{z}\left(\alpha_{0}\right)=0
\end{aligned}
$$

Furthermore, from Eqs. (10) and (17), we have

$$
\left[B, H\left(\alpha_{0}\right)\right]=2\left(\begin{array}{cc}
V_{1}\left(\alpha_{0}\right) & V_{2}\left(\alpha_{0}\right)+i V_{3}\left(\alpha_{0}\right) \\
V_{2}\left(\alpha_{0}\right)-i V_{3}\left(\alpha_{0}\right) & -V_{1}\left(\alpha_{0}\right)
\end{array}\right),
$$

where $V_{1}\left(\alpha_{0}\right)=b_{3} h_{x}\left(\alpha_{0}\right)-b_{4} h_{y}\left(\alpha_{0}\right), V_{2}\left(\alpha_{0}\right)=b_{2} h_{y}\left(\alpha_{0}\right)-b_{3} h_{z}\left(\alpha_{0}\right)$, and $V_{3}\left(\alpha_{0}\right)=$ $b_{2} h_{x}\left(\alpha_{0}\right)-b_{4} h_{z}\left(\alpha_{0}\right)$. Comparing Eq. (25) with Eq. (20), we obtain another three equations:

$$
\begin{aligned}
& b_{3} h_{x}\left(\alpha_{0}\right)-b_{4} h_{y}\left(\alpha_{0}\right)=0 \\
& b_{2} h_{y}\left(\alpha_{0}\right)-b_{3} h_{z}\left(\alpha_{0}\right)=0 \\
& b_{2} h_{x}\left(\alpha_{0}\right)-b_{4} h_{z}\left(\alpha_{0}\right)=0 .
\end{aligned}
$$

For $h_{x}, h_{y}, h_{z}$, there are six equations, Eqs.(22)-(24) and (26)-(28). If they have nonzero solutions, there must be only three independent equations, and the following constraints are required:

$$
\begin{aligned}
& \lambda_{1}=a_{3} b_{4}-a_{4} b_{3}=0 \\
& \lambda_{2}=a_{2} b_{4}-a_{4} b_{2}=0 \\
& \lambda_{3}=a_{2} b_{3}-a_{3} b_{2}=0 .
\end{aligned}
$$


However, because the matrices $A$ and $B$ do not commute with each other, from Eq. (18), we know that at least one of $\lambda_{i}(i=1,2,3)$ is nonvanishing. Thus, the only solution of $h_{x}, h_{y}, h_{z}$ is

$$
\begin{aligned}
& h_{x}\left(\alpha_{0}\right)=0, \\
& h_{y}\left(\alpha_{0}\right)=0, \\
& h_{z}\left(\alpha_{0}\right)=0,
\end{aligned}
$$

which are just the necessary constraints (11) $-(13)$ on $h_{x}(\alpha), h_{y}(\alpha)$, and $h_{z}(\alpha)$ for the occurrence of degeneracy at $\alpha=\alpha_{0}$.

From the above analysis, we conclude that the minimum requirement for a two-fold degeneracy is the non-commutation of the two representation matrices of the group, which is equivalent to the constraint of an antiunitary operator with its square being -1 . If the irreducible representation has more than two representation matrices that do not commute with each other, the constraints of the non-abelian group for the two-fold degeneracy are redundant. Thus, the protection of the other group symmetry does not contradict the protection of the HAS.

\section{Conclusion}

In summary, we proved a theorem stating that any two-fold degeneracy in a quantum system must be protected by an antiunitary operator with its square being -1 . As a result, we can infer that there is no accidental degeneracy in practice, which challenges conventional knowledge. To illustrate our conclusion, we provided an example of a two-level system. Our theory provides insight into the so-called accidental degeneracy. Furthermore, our theorem applies to not only accidental degeneracy but also nonaccidental degeneracy. We point out that, although our theorem is about two-fold degeneracy, it is straightforward to generalize it to $n$-fold degeneracy by defining $n-1$ independent antiunitary operators as in Eq. (2).

\section{Acknowledgments}

This work was supported by the National Natural Science Foundation of China under Grant Nos. 11274061 and 11504171; the Natural Science Foundation of Jiangsu Province, China under Grant No. BK20150734.

\section{References}

[1] J. von Neumann and E. Wigner, On the behavior of eigenvalues in adiabatic processes, Phys. Z. 30, 467 (1929)

[2] C. Herring, Accidental degeneracy in the energy bands of crystals, Phys. Rev. 52, $365(1937)$ 
[3] H. V. McIntosh, On Accidental Degeneracy in Classical and Quantum Mechanics, Am. J. Phys. 27, 620 (1959)

[4] V. Fock, Theory of the hydrogen atom, Phys. Z. 98, 145 (1935)

[5] S. P. Alliluev, On the relation between accidental degeneracy and hidden symmetry of a system, Soviet Phys. JETP 6, 156 (1958)

[6] F. Leyvraz, A. Frank, R. Lemus and M. V. Andrés, Accidental degeneracy in a simple quantum system: A new symmetry group for a particle in an impenetrable square-well potential, Am. J. Phys. 65, 1087 (1997)

[7] A. O. Hernández-Castillo and R. Lemus, Symmetry group of a particle in an impenetrable cubic well potential, J. Phys.: Conf. Ser. 512, 012025 (2014)

[8] J. D. Smith, Quantum mechanics of the rigid rotator, Il Nuovo Cimento B 22, 337 (1974)

[9] J. M. Hou, Hidden-symmetry-protected topological semimetals on a square lattice, Phys. Rev. Lett. 111, 130403 (2013)

[10] J. M. Hou, Moving and merging of Dirac points on a square lattice and hidden symmetry protection, Phys. Rev. B 89, 235405 (2014) 